\title{
That impish TIMP: the tissue inhibitor of metalloproteinases-3
}

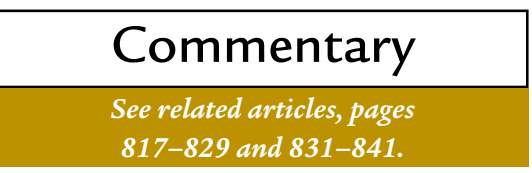

\author{
J. Frederick Woessner, Jr.
}

Department of Biochemistry R-127, University of Miami School of Medicine, PO Box 016960, Miami, Florida 33101, USA.

Phone: (305) 243-6510; Fax: (305) 243-3955; E-mail: fwoessne@med.miami.edu.

J. Clin. Invest. 108:799-800 (2001). DOI:10.1172/JCI200113709.

Our conception of the role of the ECM is rapidly changing. Early views maintained that the ECM provides a mechanical framework to hold cells together in tissues and organs. While it is still presumed that this is a major function, it is now apparent that there is considerable signaling between the ECM and the cells. All nutrients, hormones and cytokines must make their way through the ECM from blood vessels to reach the cells. The cells make contact with the matrix through sensors such as integrins on the cell surface; the ECM components profoundly affect cell behavior through such signaling (1). The glycosaminoglycan chains of proteoglycans sequester growth factors to provide a reservoir of such factors (2). Finally, cells cannot readily move from their position without disconnecting from the ECM and, frequently, degrading ECM macromolecules that lie along the path they want to traverse (3).

In order for cells to control the turnover of their ECM, to release growth factors, and to pass through the ECM when moving, they employ a wide range of proteolytic enzymes, including the plasmin system, lysosomal enzymes, and particularly the matrix metalloproteinases (MMPs) or matrixins. This latter family comprises 24 distinct genes of the human genome. There are enzymes in this family adapted to the digestion of almost every known matrix component: collagenase, gelatinases, elastase, enamelysin, and many "generalists" such as the stromelysins and matrilysins. These enzymes typically have a large propeptide containing cysteine, a catalytic domain with zinc at the active center, and a hemopexin-like domain. Additional domains may include fibronectin-like, collagen-like, and transmembrane domains (4).

\section{Role of TIMPs}

It is critical to maintain control of extracellular proteolytic activity so that untrammeled digestion does not destroy critical tissues. Most MMPs are made only upon demand and in low levels; they are secreted as proenzymes in which a cysteine residue of the propeptide binds to and inactivates the active-site zinc. Most importantly, there is a group of five TIMPs (tissue inhibitors of metalloproteinases) that are each capable of inhibiting almost every member of the MMP family. The TIMPs are small proteins of about 21,000 Da that contain two domains: $\mathrm{N}$-terminal and C-terminal. Each domain contains three disulfide bridges, making the TIMPs quite stable. Most of the biological functions discovered so far reside in the $\mathrm{N}$-terminal domain of about 125 residues (5). Normally, the TIMPs are in delicate balance with the MMPs and matrix is digested in a highly regulated fashion. However, there are many disease processes in which MMP levels are elevated without a concomitant increase in TIMPs, leading to an imbalance and the resultant destruction of tissues. Some wellknown examples include the loss of cartilage matrix in osteo- and rheumatoid arthritis, the rupture of the plaque cap in atherosclerosis, and the invasion and metastasis of tumor cells (6).

A surprising feature of the TIMPs is their multiplicity of biological roles. Early observations of an erythroidpotentiating factor led to purification and cloning. Shortly afterwards, TIMP1 was cloned and sequenced and found to be the identical protein (5). Subsequent work has amply demonstrated the growth effects of TIMP-1 and TIMP-2 $(5,6)$. TIMP-2 has a further role, not completely understood, in the binding of progelatinase A (MMP-2) to membrane-type matrix metalloproteinase-1 (MMP-14) as a prerequisite to the activation of MMP-2 to its active form. TIMP-1 and TIMP-2 also bind to gelatinases B (MMP-9) and A, respectively, when these are in their proen- zyme form, an interaction in which the TIMPs bind to the hemopexin domain of the MMPs, not to the active center, which is concealed (5).

\section{Unique properties of TIMP-3}

TIMP-3 has further unexpected properties. First, it is the only TIMP that binds firmly to the ECM. In fact, it was first discovered as an ECMbound molecule produced by cells undergoing transformation (7). This binding is now believed to be due to interaction of the $\mathrm{N}$-terminal domain with heparan sulfate and chondroitin sulfate chains of cell surface or secreted proteoglycans (8). Through these interactions, TIMP-3 is localized where it can inhibit sheddases or regulate movement through the basement membrane and stroma. TIMP-3 not only inhibits MMPs but is also capable of inhibiting members of two groups within the adamalysin family: the ADAMs (a $\underline{\text { disintegrin and } \underline{\text { a }} \text { met- }}$ alloproteinase domain) and the ADAMTSs (ADAM with thrombospondin-like repeats). It can inhibit TACE (TNF- $\alpha$-cleaving enzyme, ADAM 17, ref. 9), ADAM 10 (10), and ADAM 12S (11); it also inhibits the shedding of IL-6 (12), L-selectin (13), and syndecans 1 and 4 (14), which are thought to be mediated by ADAMtype proteases. TIMP-3 can also inhibit members 4 and 5 of the ADAMTS group, enzymes that are responsible for aggrecan degradation in cartilage (15). TIMP-3 is the only TIMP known to be related to a disease: mutation of certain cysteine residues to serine results in deposition of excessive amounts of TIMP-3 in Bruch's membrane, producing early blindness, a condition known as Sorsby fundus dystrophy (16).

Perhaps the most interesting effect of TIMP-3 is its ability to initiate cell apoptosis. TIMP-1 and TIMP-2, by contrast, suppress apoptosis. Thus, 
overexpression of TIMP-3 induces apoptosis in various cancer cell lines and in rat vascular smooth muscle, perhaps by inhibiting the shedding of the TNF- $\alpha$ receptor from the cell surface (17-19). These apoptotic effects cannot be mimicked by addition of synthetic MMP inhibitors; however, it has been suggested that these inhibitors lack the correct specificity (20). The N-terminal domain of TIMP-3 is responsible for the apoptotic effect, and mutation of the critical Cys1 residue destroys the effect, indicating that the TIMP-3 must be in a form that can inhibit proteases (20).

\section{The TIMP-3 knockout mouse}

Until now, all of the available information on TIMP-3 derived from enzyme assays or cell culture work, but in this issue of the JCI, two papers from the Khokha group $(21,22)$ examine the effects of knocking out the gene for TIMP-3 to understand the protein's effects in vivo. In the first paper (21), it is shown that the null mouse survives only about half as long as the wild-type. Striking effects are seen in the lungs, which show enlarged air spaces at 2 weeks of age that progress with age. Aged animals exhibit a decreased amount of lung collagen, and the uptake of gases is severely impaired. However, there is no inflammatory cell infiltration or fibrosis. It is thought that the increased MMP activity, demonstrated by several means, in the face of no increase of TIMP-1 and - 2 and the absence of -3 , leads to a net destruction of lung ECM. The classical view that an imbalance of MMP and TIMP leads to collagen destruction appears to account for most of the observations.

The findings of the second paper (22) are more paradoxical. Despite previous evidence that overexpression of TIMP-3 leads to apoptosis, the involuting mammary glands of the Timp-3 $3^{-/-}$mice show a peak of mammary epithelial cell apoptosis at 1 day after weaning, compared with 3 days in wild-type mice. This corresponds to the first wave of apoptosis involving the differentiated epithelial cells (23). Therefore, a deficiency of TIMP-3 is proapoptotic, whereas in other situations overexpression leads to apoptosis. One possibility is that the response is biphasic, so that absence of TIMP-3 or excessive levels are both proapoptotic, whereas normal levels have no effect. However, it seems more likely, as the authors suggest, that mammary epithelial cells in vivo may behave differently from cancer cells in vitro. There is evidence in the null mouse that MMP-2 becomes activated early (TIMP-3 might normally block activation by MMP-14) and fibronectin fragments are released earlier, indicating ECM breakdown. These untimely events are suppressed by addition of exogenous TIMP-3 in pellet implants and by use of synthetic MMP inhibitors. The results do not give a clear picture of which proteases might be involved MMPs, ADAMs, or ADAMTSs.

These first reports from the Timp-3null mouse are quite exciting and suggest that many more effects of TIMP-3 remain to be uncovered. In particular, it may be possible to determine the consequences of failure to inhibit MMPs and, especially, the ADAMs and ADAMTSs, which so far seem to lack other specific biological inhibitors. More importantly, it should be possible to trace out the pathways by which TIMP-3 is able to regulate apoptosis and to influence the cell cycle.

1. Giancotti, F.G., and Ruoslahti, E. 1999. Integrin signaling. Science. 285:1028-1032.

2. Park, P.W., et al. 2000. Cell surface heparan sulfate proteoglycans: selective regulators of ligand-receptor encounters. J. Biol. Chem. 275:29923-29926.

3. Murphy, G., and Gavrilovic, J. 1999. Proteolysis and cell migration: creating a path? Curr. Opin. Cell Biol. 11:614-621.

4. Nagase, H., and Woessner, J.F. 1999. Matrix metalloproteinases. J. Biol. Chem. 274:21491-21494.

5. Brew, K., et al. 2000. Tissue inhibitors of metalloproteinases: evolution, structure and function. Biochim. Biophys. Acta. 1477:267-283.

6. Gomez, D.E., et al. 1997. Tissue inhibitors of metalloproteinases: structure, regulation and biolog- ical function. Eur. J. Cell Biol. 74:111-122.

7. Blenis, J., and Hawkes, S.P. 1984. Characterization of a transformation-sensitive protein in the extracellular matrix of chicken embryo fibroblasts. $J$ Biol. Chem. 259:11563-11570.

8. Yu, W.H., et al. 2000. TIMP-3 binds to sulfated glycosaminoglycans of the extracellular matrix. $J$ Biol. Chem. 275:31226-31232.

9. Amour, A., et al. 1998. TNF-alpha converting enzyme (TACE) is inhibited by TIMP-3. FEBS Lett. 435:39-44.

10. Amour, A., et al. 2000. The in vitro activity of ADAM-10 is inhibited by TIMP-1 and TIMP-3 FEBS Lett. 473:275-279.

11. Loechel, F., et al. 2000. ADAM 12-S cleaves IGFBP3 and IGFBP-5 and is inhibited by TIMP-3 Biochem. Biophys. Res. Commun. 278:511-515.

12. Hargreaves, P.G., et al. 1998. Human myeloma cells shed the interleukin- 6 receptor: inhibition by tissue inhibitor of metalloproteinase- 3 and hydroxamate-based metalloproteinase inhibitor. Br. J. Haematol. 101:694-702.

13. Borland, G., et al. 1999. Tissue inhibitor of metalloproteinases- 3 inhibits shedding of L-selectin from leukocytes. J. Biol. Chem. 274:2810-2815.

14. Fitzgerald, M.L., et al. 2000. Shedding of syndecan- 1 and -4 ectodomains is regulated by multiple signaling pathways and mediated by a TIMP-3sensitive metalloproteinase. J. Cell Biol. 148:811-824.

15. Kashiwagi, M., et al. 2001. TIMP-3 is a potent inhibitor of aggrecanase 1 (ADAM-TS4) and aggrecanase 2 (ADAM-TS5). J. Biol. Chem. 276:12501-12504.

16. Felbor, U. 1995. A novel Ser156Cys mutation in the tissue inhibitor of metalloproteinases-3 (TIMP3) in Sorsby's fundus dystrophy with unusual clinical features. Hum. Mol. Genet. 4:2415-2416.

17. Ahonen, M., et al. 1998. Adenovirus-mediated gene delivery of tissue inhibitor of metalloproteinases-3 inhibits invasion and induces apoptosis in melanoma cells. Cancer Res. 58:2310-2315.

18. Baker, A.H., et al. 1998. Divergent effects of tissue inhibitor of metalloproteinase- $1,-2$, or -3 overexpression on rat vascular smooth muscle cell invasion, proliferation, and death in vitro: TIMP-3 promotes apoptosis. J. Clin. Invest. 101:1478-1487.

19. Smith, M.R., et al. 1997. TIMP-3 induces cell death by stabilizing TNF-alpha receptors on the surface of human colon carcinoma cells. Cytokine. 9:770-780

20. Bond, P., et al. 2000. Localization of the death domain of tissue inhibitor of metalloproteinase3 to the $\mathrm{N}$ terminus: metalloproteinase inhibition is associated with proapoptotic activity. J. Biol. Chem. 275:41358-41363.

21. Leco, K.J., et al. 2001. Spontaneous air space enlargement in the lungs of mice lacking tissue inhibitor of metalloproteinases-3 (TIMP-3). J. Clin. Invest. 108:817-829.

22. Fata, J.E., et al. 2001. Accelerated apoptosis in the Timp-3-deficient mammary gland. J. Clin. Invest. 108:831-841.

23. Lund, L.R., et al. 1996. Two distinct phases of apoptosis in mammary gland involution: proteinase-independent and -dependent pathways. Development. 122:181-193. 